Renate Müller u. a.: Musikalische und mediale Selbstsozialisation

Peter Imort: „Musikalische und mediale Selbstsozialișation." Eine Diskussion über Herausforderungen und Perspektiven der Theorie
* Horst Niesyto: Kritische Anmerkungen zum Konzept "medialer Selbstsozialisation"

Manfred Pirner: Selbstsozialisation - zur pädagogischen Tragfähigkeit eines soziologischen Konzepts

\section{Musikalische und mediale Selbstsozialisation}

\section{Renate Müller, Stefanie Rhein, PATRICK Glogner}

Der folgende Beitrag gliedert sich in drei Teile: Er beginnt mit einer Zusammenfassung unseres Beitrags, der der Diskussion des IZMM zu Grunde lag. Es folgt das Abstract der nächsten von uns geplanten Arbeit. Der Beitrag schließt mit einer Übersicht über unsere Publikationen zur musikalischen und medialen Selbstsozialisation.

1. Z usammenfassung: D as Konzept musikalischer und medialer Selbstsozialisation - widersprüchlich, trivial, überflüssig? In: $\mathrm{H}$ offmann, Dacmar/ Merk ens, H ans (H g.) (2004): Jugendsoziologische Sozialisationstheorie. Impulse fur die Jugendforschung. W einheim und M ünchen: Juventa, 237-252.

D as Konzept der musikalischen und medialen Selbstsozialisation wird seit mehr als einem Jahrzehnt als Weiterentwicklung traditioneller Sozialisationstheorien betrachtet. Die Berliner Tagung „Sozialisationstheorien auf dem Prüfstand" nahmen zum Anlass, den Selbstsozialisationsansatz auf „Herz und Nieren“ zu prüfen: Ist das Konzept womöglich widersprüchlich und trivial und somit fragwürdig und überflüssig? D ie Auseinandersetzung mit dieser Frage erfolgt in drei Schritten:

1. Das Konzept der musikalischen und medialen Selbstsozialisation wird dargestellt:

a) Zentrale Aussagen, b) verwandte und

c) kontrastierende theoretische Ansätze,

d) empirische Überprüfungen.

2. Dilemmata des Konzepts werden im Hinblick auf dessen Widersprüchlichkeit und Trivialität diskutiert.

3. Eine Perspektive wird entwickelt, aus deren Sicht der Selbstsozialisationsansatz als nicht überflüssig erscheint.

\section{$\mathrm{Zu} 1$.}

a) Das Konzept musikalischer und medialer Selbstsozialisation basiert auf der Idee "des produktiv realitätsverarbeitenden Subjekts" (Hurrelmann) und betont die Eigenleistung des Individuums im Sozialisationsprozess, die mit der Individualisierung und der Ent- standardisierung von Lebensläufen erforderlich wird. Musikalische und mediale Selbstsozialisation vollziehen sich durch das Mitgliedwerden in selbstgewählten Kulturen, wobei die gewählte Symbolwelt angeeignet, der entsprechende Lebensstil übernommen und musik- und medienkulturelle Kompetenzen erworben werden; dadurch werden Zugehörigkeiten und Abgrenzungen definiert und Identitäten konstruiert.

b) Der Selbstsozialisationsansatz knüpft an den Symbolischen Interaktionismus und den Cultural Studies-Ansatz an und steht solchen Konzepten nahe wie dem des populär-kulturellen Kapitals, dem der parasozialen Interaktion, dem der Wahlnachbarschaften, denen der emotions- und sozialästhetischen Umgehensweisen mit Kultur.

c) D esgleichen werden Ansätze angeführt, die der Selbstsozialisationansatz kritisiert und deren "blinde Flecken" er unter die Lupe nimmt: insbesondere die kulturkritische musiksoziologische Perspektive Adomos, in deren Zentrum die Vermassungshypothese und die Doktrin stehen, dass die Umgehensweise dem jeweiligen kulturellen Objekt inhärent sei. Eng verknüpft mit dieser D oktrin ist die normative Hierarchisierung kultureller Objekte (z. B. der Hoch- vs. der Populärkultur) sowie der vermeintlich jeweils adäquaten Umgehensweisen mit Kultur (z. B. aktiv vs. passiv) und, nicht zuletzt, der jeweiligen Rezipienten. Bourdieus vehemente Kritik der gesellschaftlichen Urteilskraft kritisiert genau diese Doktrin als Reproduzentin gesellschaftlicher Ungleichheit, da sie im vermeintlich autonomen Kunstobjekt die Umgehensweisen ansiedelt, die doch in Wirklichkeit auf sozialisierten Codes basieren. Diese Kritik greift insofern zu kurz, als sie die sozialisatorisch vermittelte Kodienung ästhetischer O bjekte der Populärkultur ausdrücklich ausschließt. Somit besteht das kritische theoretische wie empirische Potenzial der Selbstsozialisationstheorie darin, ästhetische Objekte der Populärkultur für kodiert zu halten, Aneignungsweisen populärkultureller Codes für notwendig und existent zu erklären und die bedeutsame Rolle 
dieser Aneignungsweisen bei der Sozialisation und Identitätskonstruktion zu betonen. D arüber hinaus kritisiert die Selbstsozialisationstheorie die stereotype normative Hierarchisierung von Kulturen, von Umgehensweisen mit Kulturen sowie von Kulturrezipienten.

d) D as Selbstsozialisationskonzept hat sich in der Erforschung des sozialen Gebrauchs nicht nur von Musik und Medien, sondem von kulturellen Symbolsystemen generell als fruchtbar, das heißt u. a. als erklärungskräftig erwiesen. Dabei konnte beispielsweise in Untersuchungen zum Umgehen mit der Dr.-Sommer-Beratung in BRAVO, zur fanspezifischen Nutzung populärmusikalischer Angebote, zur Rezeption jugendlicher Videoproduktionen sowie zu gruppenspezifischen Sprechstilen ausländischer Jugendlicher bestätigt werden, dass Jugendliche beim Umgehen mit kulturellen Symbolsystemen ihre Identität konstruieren, sich selbst sozialisieren, parasozial interagieren, populärkulturelles Kapital anhäufen und Entwicklungsaufgaben lösen.

\section{$\mathrm{Zu} 2$.}

Der Beitrag setzt sich mit den Dilemmata a) der Dichotomisierung von Hoch- und Populärkultur, b) der Fokussierung auf Jugendund Populärkultur, c) der Dichotomisierung von Selbst- und Fremdsozialisation, d) der Trivialität (Sozialisation ist immer Selbstsozialisation) und e) der Verschleierung sozialer Ungleichheit auseinander. Thesenartig seien die Ergebnisse dieser Auseinandersetzung wiedergegeben:

a) Der Ansatz wendet sich gegen die Hierarchisierung, nicht jedoch gegen die begriffliche Unterscheidung von Hoch- und Populärkultur. Die in der Selbstsozialisationsperspektive bisher durchgeführten Forschungsarbeiten nehmen gerade deswegen populärkulturelle Selbstsozialisationsprozesse in den Blick, weil diese von den kritisierten Perspektiven marginalisiert, ignoriert oder abgewertet werden.

b) Selbstsozialisierer gibt es überall, nicht nur in der Jugend- und Populärkultur. So kann gezeigt werden, dass beispielsweise JugendMusiziert-Gewinner, Erwachsene in der Berufssozialisation und erwachsene Konzert- und Kinopublika sich selbst sozialisieren.

c) Selbstsozialisation ist streng genommen nie reine bzw. ausschließliche "Selbst"Sozialisation, denn Selbstsozialisation braucht Anregungen von außen, z. B. durch Sozialisationsinstanzen. Fremdsozialisation ist streng genommen nie reine bzw. ausschließliche "Fremd“-Sozialisation, denn intentionale Erziehung braucht die Akzeptanz durch das zu sozialisierende Individuum.

d) D as Verhältnis von Fremd- und Selbstsozialisation ist vorstellbar als ein Kontinuum, dessen Endpunkte (Fremdsozialisation Selbstsozialisation) nur als Konstrukte existieren: Es gibt weder reine Selbst- noch reine Fremdsozialisation. Verschiedene soziokulturelle Kontexte bergen jeweils unterschiedliche Selbst- und Fremdsozialisationspotenziale.

e) Da es offenbar Bedingungen gibt, unter denen Sozialisation kaum Selbstsozialisation sein kann, wird es notwendig, Bedingungen zu spezifizieren, unter denen Selbstsozialisation mehr oder weniger möglich ist.

$\mathrm{Zu} 3$.

Der Selbstsozialisationsansatz kann u. E. nur fruchtbar sein, wenn Selbstsozialisation nicht als die einzige Sozialisationsmöglichkeit in der gegenwärtigen $\mathrm{G}$ esellschaft gedacht wird. $\mathrm{Zu}$ fragen ist vor dem Hintergrund der skizzierten Dilemmata und der diskutierten Lösungsvorschläge vielmehr nach der Kontextabhängigkeit von Selbstsozialisationspotenzialen verschiedener Kulturen und ihrer Vermittlungsund Gebrauchsinstitutionen. Hier liegen die zukünftigen Aufgaben der Weiterentwicklung des Selbstsozialisationsansatzes, der keineswegs für überflüssig erachtet wird, so lange soziale G ebrauchsweisen populärer Kultur in der kritisierten Weise marginalisiert werden.

2. A usblick: V ortragsabstract fur die 20. Jahrestagung der D eutschen $\mathrm{G}$ esellschatt fur M usikpsychologie 2004 in Paderborn (3.-5. September 2004) "M usik alisches L ernen in der Schule und anderswo"

Die Theorie musikalischer Selbstsozialisation: Elf Jahre ... und ein bisschen weiser?

\section{Hintergrund}

Auf der 9. Jahrestagung der D GM in Münster 1993 sowie im 11. Jahrbuch Musikpsychologie wurde die Theorie musikalischer Selbstsozialisation vorgestellt: Musikalische Selbstsozialisation ist das Mitgliedwerden in selbst gewählten Musikkulturen, wobei die gewählte Symbolwelt angeeignet, der entsprechende Lebensstil übernommen und rezeptive wie produktive musikkulturelle Kompetenzen selbst organisiert erworben werden; dadurch werden Zugehörigkeiten und Abgrenzungen definiert und Identitäten konstruiert. Seitdem wurde das Konzept musikalischer Selbstsozialisation im Rahmen empirischer Untersuchungen und theoretischer Diskurse weiterentwickelt und zur Erklärung des Umgehens Jugendlicher mit Musik angewandt. 


\section{Ziele und Methoden}

D as theoretische und empirische Potenzial der Theorie musikalischer Selbstsozialisation wird diskutiert: Zentrale Aussagen der Theorie, ihre Bezüge zu und Konfrontation mit anderen Theorien sowie theoretische Probleme (Widersprüchlichkeit, Trivialität, „blinde Flecken") werden skizziert. Empirische Überprüfungen werden exemplarisch dargestellt. Die Ausarbeitung theoretischer Konstrukte (z. B. Globalisierung, kulturelles Gedächtnis, symbolische Exklusion) im Zusammenhang der aktuellen Jugendkulturforschung wird vorgestellt.

\section{Ergebnisse}

Die Theorie musikalischer Selbstsozialisation scheint empirisch und theoretisch fruchtbare Konstrukte zum Umgehen Jugendlicher mit Musik zu enthalten: Jugendliche konstruieren und präsentieren Identität mit Musik. Sie erlernen audiovisuelle Symbolsprachen, die sie zur interkulturellen Kommunikation befähigen. Jugendliche eignen sich globale Musikkulturen an und beziehen sie in lokalen Praktiken auf ihr Leben. Autodidaktisch und kooperativ erwerben sie musikkulturelle Kompetenzen, mittels derer sie sich soziokulturell verorten. Jugendliche gebrauchen Musik, um Probleme zu bearbeiten, die sich geschlechts-, bildungs- und schichtspezifisch sowie nach ethnischer Zugehörigkeit je unterschiedlich stellen.

\section{Schlussfolgenungen}

Zukünftige theoretische und empirische Aufgaben zur Elaboration der Theorie werden genannt. Vorrangig erscheint die Spezifikation von Bedingungen, unter denen Chancen zur musikalischen Selbstsozialisation mehr oder weniger gegeben sind.

\section{Unsere Publikationen zur Selbstsozialisation}

Behne, Klaus-Ernst/ Müller, Renate (1996): Rezeption von Videoclips - Musikrezeption. Eine vergleichende Pilotstudie zur musikalischen Sozialisation. In: Rundfunk und Fernsehen 44:3, 365-380.

Glogner, Patrick (2000): Selbstsozialisation und Identitätskonstruktion mit der Dr.Sommer-Seite. Eine Befragung mit dem MultiMedia-Computer zur Rezeption der Jugendzeitschrift BRAVO. In: deutsche jugend. Heft 7/ 8, 318-326.
Glogner, Patrick (2002b): Sozial-ästhetische Umgehensweisen mit Filmen. Ausgewählte Ergebnisse einer empirischen Untersuchung von Kinobesuchern. In: Heinrichs, Werner/ Klein, Armin (Hg.): D eutsches Jahrbuch für Kulturmanagement 2001. Band 5. BadenBaden: Nomos, 91-111.

Müller, Renate (1994): Oi!-Musik und fremdenfeindliche G ewalt: Zur kulturellen Identität von Skinheads (Teil 1)/ Was können wir tun? (Teil 2) In: Musik \& Bildung, 26: 3, 4650/ 26: 4, 44-48.

Müller, Renate (1995): Selbstsozialisation. Eine Theorie lebenslangen musikalischen Lernens. In: Jahrbuch Musikpsychologie, 11, 63-75.

Müller, Renate (1996a): Geschlechtsspezifisches Umgehen mit Videoclips: Erleben Mädchen Videoclips anders? In: Musikpädagogische Forschung, Bd. 17, hg. von Hermann J. Kaiser, Essen: Die Blaue Eule, 7393.

Müller, Renate (1996b): Skinheads and Violence: Blame it on Oil-Music? In: MusicMedicine Vol II, ed. by Pratt, Rosalie Rebello/ Spintge, Ralph. Saint Louis: MMB Music, 117-128.

Müller, Renate (1998): Musikalische Sozialisation und Identität. Ergebnisse einer computerunterstützten Befragung mit dem klingenden Fragebogen. In: Entwicklung und Sozialisation aus musikpädagogischer Perspektive, Musikpädagogische Forschung, Bd. 19, hg. von Mechthild von Schönebeck, Essen: Die Blaue Eule, 57-74.

Müller, Renate (1999a): Musikalische Selbstsozialisation. In: J. Fromme, S. Kommer, J. Mansel, K.-P. Treumann, (Hg.): Selbstsozialisation, Kinderkultur und Mediennutzung. O pladen: Leske+Budrich, 113-125.

Müller, Renate (1999b): Selbstsozialisation Jugendlicher durch Musik und Medien. In: Bertelsmann Briefe, Heft 142, 12-15.

Müller, Renate (2000): Identitätskonstruktion und Selbstsozialisation Jugendlicher durch Mitgliedschaft in musikalischen Jugendkulturen. In: Kathrin Eberl und Wolfgang Ruf (Hg.): Musikkonzepte - Konzepte der Musikwissenschaft, Kongressbericht Halle 1998, Bd. 1. Halle: Bärenreiter, 299-306.

Müller, Renate (2002a): Perspectives from the Sociology of Music. In: R. Colwell, C. Richardson (Hg.): The New Handbook of Research on Music Teaching and Learning. New York: Oxford University Press, 584603. 
Müller, Renate (2002b): Präsentative Methoden zur Erforschung des Umgehens Jugendlicher mit Musik und Medien. Der MultiMediaComputer als Erhebungsinstrument in der Jugendforschung. In: Müller, Renate/ Glogner, Patrick/ Rhein, Stefanie/ Heim, Jens (Hg.): Wozu Jugendliche Musik und Medien gebrauchen. Jugendliche Identität und musikalische und mediale Geschmacksbildung. Weinheim, München: Juventa, 242-255. Müller, Renate (2003): Audiovisuelle Fragebögen zur Messung der Attraktivi-tät jugendlicher Videoproduktionen. In: Niesyto, Horst (Hg.): VideoCulture - Video und interkulturelle Kommunikation. München: kopaed, 283-315.

Müller, Renate (2004a): Musiksoziologische Grundlagen. In: Wickel, Hans Hermann/ Hartogh, Theo (Hg.). Handbuch Musik in der Sozialen Arbeit. Weinheim und München: Juventa (im D ruck).

Müller, Renate (2004b): Zur Bedeutung von Musik für Jugendliche. In: merz 48:2 (im D ruck).

Müller, Renate/ Behne, Klaus-Emst (1996): Wahrnehmung und Nutzung von Videoclips - Eine vergleichende Pilotstudie zur musikalischen Sozialisation, Forschungsbericht Nr. 6, Institut für Musikpädagogische Forschung an der Hochschule für Musik und Theater Hannover.

Müller, Renate/ Dongus, Nadine/ Ebert, Sabine/ Glogner, Patrick/ Kreutle, Andreas (1999): Identitätskonstruktion mit Medien und Musik. Ein empirisches Forschungsprogramm mit MultiMedia-Fragebögen. In: medien praktisch, 22:1, 26-30.

Müller, Renate, Glogner, Patrick, Rhein, Stefanie, Heim, Jens (2002): Zum sozialen Gebrauch von Musik und Medien durch Jugendliche. Überlegungen im Lichte kultursoziologischer Theorien. In: Müller, Renate/ Glogner, Patrick/ Rhein, Stefanie/ Heim, Jens (Hg.): Wozu Jugendliche Musik und Medien gebrauchen. Jugendliche Identität und musikalische und mediale Geschmacksbildung. Weinheim, München: Juventa, 9-26.

Müller, Renate/ Rhein, Stefanie/ Glogner, Patrick (2004): Das Konzept musikalischer und medialer Selbstsozialisation - widersprüchlich, trivial, überflüssig? In: Hoffmann, Dagmar/ Merkens, Hans (Hrsg.). Jugendsoziologische Sozialisationstheorie. Impulse für die Jugendforschung. Weinheim und München: Juventa, 237-252.
Rhein, Stefanie (2000): „Being a Fan is More than That" - Fan-Specific Involvement with Music. In: the world of music, 42:1, 95-109.

Rhein, Stefanie (2000): Teenie-Fans: Stiefkinder der Populärmusikforschung. Eine Befragung Jugendlicher am MultiMediaComputer über ihre Nutzung fankultureller Angebote. In: Deutsches Jahrbuch für Kulturmanagement 1999, hg. v. Werner Heinrichs u. Armin Klein, Baden-Baden: Nomos, 165-194.

In Vorbereitung

Müller, Renate/ Glogner, Patrick/ Rhein, Stefanie: Die Theorie musikalischer Selbstsozialisation: Elf Jahre ... und ein bisschen weiser?

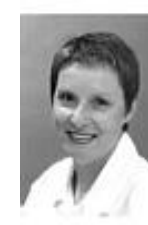

Prof. Dr. Renate Müller

Professorin für Erziehungs- und Kultursoziologie an der PH Ludwigsburg

A rbeitsschwerpunk te:

Umgehen von Jugendlichen mit Musik und Medien, empirische präsentative Methoden, computerunterstützte audiovisuelle Forschungsinstrumente, musik- und mediensoziologische, musik- und medienpädagogische Grundlagenforschung und angewandte Forschung, Unterrichtsmodelle zum Rock- und Poptanz im Musikunterricht.

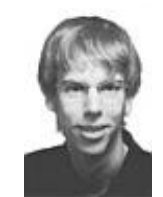

Patrick Glogner, M.A.

Wiss. Mitarbeiter am Institut für Kulturmanagement an der $\mathrm{PH}$ Ludwigsburg

A rbeitsschwerpunk te:

Filmpublika und ihre Ungehensweise mit Filmen; empirische Untersuchung zur soziologischen Differenzierung von Kinobesuchern

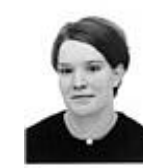

Stefanie Rhein, M.A.

Tutorin und wiss. Hilfskraft an der Musiksoziologischen Forschungsstelle der PH Ludwigsburg A rbeitsschwerpunkt:

Musikfans und die fanspezifische Nutzung populärmusikalischer Angebote 


\section{„Musikalische und mediale Selbstsozialisation" Eine Dis- kussion über Herausforderun- gen und Perspektiven der Theorie}

\section{PETER IMORT}

Im Januar 2004 trafen sich IZMM-Mitglieder in der Pädagogischen Hochschule Ludwigsburg zur D iskussion der Theorie der musikalischen und medialen Selbstsozialisation. Den Impuls zum anregenden Disput lieferte der Beitrag von Renate Müller, Stefanie Rhein und Patrick Glogner: „D as Konzept musikalischer und medialer Selbstsozialisation - widersprüchlich, trivial, überflüssig?"

Nach einer Runde kürzerer Eingangsstatements von Peter Holzwarth, Petra ReinhardHauck, Horst Niesyto, Peter Imort, Manfred Pirner sowie den anwesenden Autorinnen und dem Autor begann eine gut zweistündige, intensive und kontroverse Diskussion der Thematik. Die folgende Darstellung folgt weniger der Chronologie des $\mathrm{G}$ esprächs als seinen thematischen Schwerpunkten aus meiner Perspektive. Meine verkürzte Charakteristik der Diskussion soll die Lektüre des Basistextes nicht ersetzen, sondern neugierig auf das Papier machen, das von der Runde als prägnant, klar gegliedert, auf der Höhe ihrer Theoriebildung und begrifflich sehr präzise argumentierend charakterisiert wurde.

\section{Selbstsozialisation - Eine neue Theorie mit alten (Un-)bekann- ten?}

Zweifellos sind Individuen heute viel stärker gefordert, darüber nachzudenken, wie sie ihr Leben gestalten, und entsprechend zu handeln. D ie Vorstellung von weitgehend selbstgesteuerten Sozialisationsprozessen, in denen Individuen aus einem Pool von Angeboten aktiv wählen, Präferenzen setzen und Identitäten ausbalancieren, forciert Fragestellungen, die das $\mathrm{G}$ ewicht der klassischen, institutionalisierten Sozialisationsagenturen (Familie, Schule, Betrieb usw.) insbesondere gegenüber Peers und speziell gegenüber Medien neu austarieren. Diese Neubewertung hat nicht nur dazu geführt, dass die Untersuchung der klassischen Sozialisationsagenturen in der Empirie an Bedeutung verloren hat. Die stärkere Beschäftigung mit dem Subjektbegriff hat außerdem zu einer Reihe von Theorien geführt, die den Subjektbegniff stärker in den Vordergrund stellen. D as Präfix „Selbst“ fungiert „als Krücke, diesen paradigmatischen Wandel anzudeuten" (H. Niesyto).
Die Autoren vertreten Selbstsozialisation nicht als Standpunkt einer neuen soziologischen Theorie. Renate Müller betont vielmehr die Nähe zu Ansätzen klassischer Sozialisationstheorien, z. B. dem Symbolischen Interaktionismus. Daraus wird gewissermaßen auf die Spitze formuliert, um den Blick für viele Dinge zu eröffnen, die in Musikpädagogik und Musikwissenschaft bislang so nicht oder zu wenig gesehen wurden. Man kann so weit gehen, Selbstsozialisation als Gegenpol zur Massifikationshypothese der Kritischen Theorie besonders in der Adorno-Nachfolge zu sehen. Die Indizien sprechen für ein hohes Maß an Fremdbestimmung, Manipulation und Entfremdung, auf der anderen Seite sind ebenso deutlich Beobachtungen zu machen, die die aktive, kulturelle Selbststeuerung des einzelnen Jugendlichen hervorheben. Wie kann man den Sachverhalt mit dem Pool an Möglichkeiten heute fassen, differenzierter und nicht so negativ wertend? (Peter Holzwarth)

Selbstsozialisation erscheint als eine soziologische Theorie. Jedoch weisen Termini wie z.B. „selbstgesteuertes Lemen“ oder "selbstorganisierte Bildungsarbeit" auf Bezugspunkte und Parallelen hin, die außerhalb der Soziologie liegen. Manfred Pimer weist auf die Beheimatung der Begriffe "Subjekt", "Subjektorientierung" und „Autonomie des Subjekts" in der Philosophie hin, die dann auch in andere Bereiche übernommen wurde, u. a. auch in die Pädagogik bzw. die Didaktik. Auf der pädagogischen Ebene lässt sich Selbstsozialisation möglicherweise als ein kritisch-emanzipativer Prozess beschreiben. Insofern könnte man die Frage stellen, inwiefern die Theorie der Selbstsozialisation als Instrument der Beschreibung und Analyse hilfreich im Vergleich zu einer fundierten Bildungstheorie ist.

Die Frage nach dem Verhältnis von Sozialisation und Selbstsozialisation wird sehr unterschiedlich und kontrovers diskutiert. Horst Niesyto fragt, ob man angesichts der Tatsache, dass das Subjekt größeres G ewicht hat, wirklich von "Selbst"-"sozialisation" sinnvoll sprechen kann, da im Sozialisationsbegriff schon immer das Verhältnis von Individuum und Gesellschaft angelegt ist. In system- und strukturfunktionalen Theorien ist die leitende Fragestellung die der Anpassung von Individuen an gesellschaftliche Normen und Wertesysteme. D er Bezug liegt außerhalb des Individuums, das Individuum ist Teil der Gesellschaft. Es gibt wirksame äußere Sozialisationsagenturen, gesellschaftliche Normen und Sanktionen, aber die Individuen entscheiden immer selbst, inwieweit und in welcher Intensität sie folgen. Wenn man so will, ist das der klassische sozial- 
integrative Ansatz: Die Individuen sollen sich den vorgegebenen Normen und Werten anpassen. Allerdings ist - und das ist kritisch zu hinterfragen - in system- und strukturfunktionalen Theorien der "eigene Teil" tendenziell passiv angelegt. Dennoch erscheint die Silbe "selbst-" in diesem Zusammenhang irreführend und läuft Gefahr, Missverständnisse über den Sozialisationsbegriff zu evozieren. G erade weil es unterschiedliche Verständnisse, Interessen und Kräfte gibt, die den Sozialisationsbegniff füllen wollen und den Fokus, durchaus im Sinne von Selbstsozialisation, beispielsweise auf Erziehung, Integration oder Persönlichkeitsbildung setzen, bestehen Zweifel an der Verwendung des Begriffs.

Ein weiteres Fragezeichen betrifft den aktuellen Forschungszusammenhang der Theorie. Wie geht die Diskussion außerhalb des hiesigen K reises weiter? Zu klären ist, warum der Begriff z. B. von der G ruppe Bielefelder Soziologen um Jürgen Zinnecker, die ihn selbst angestoßen und 1998 eine nationale Konferenz organisiert haben, gegenwärtig nicht weiter verfolgt wird. $\mathrm{Ob}$ man so weit gehen muss, den Begriff aus diesen G ründen fallen zu lassen, ist für die anderen Diskussionsteilnehmer fraglich. Selbstsozialisation wirft viele Fragen auf, bringt andererseits vieles auf den Punkt. Wichtig ist, die Theorie kritisch zu hinterfragen und den "Mehrwert zu eruieren, der in der Erweiterung der Sozialisationsperspektive steckt" (Manfred Pimer).

Wohin entwickelt sich die Theorie der „Selbstsozialisation“? Die Skizzierung weiterer Diskussionspunkte zeichnet einige Perspektiven und Problemkreise auf.

\section{Selbstsozialisation - Fremdsoziali- sation}

In dem Artikel wird die begriffliche Dichotomie „Selbstsozialisation - Fremdsozialisation" aufgebaut. Dieses sorgte für intensiven Gesprächsstoff und wurde $z$. T. als problematisch angesehen. Stefanie Rhein berichtet, dass im Autorenkreis während der Konzeption des Artikels lange über die Verhältnisbestimmung von Selbst- und Fremdsozialisation diskutiert wurde, die Problematik wurde gesehen und die Unterscheidung aus verschiedenen $G$ ründen nicht für günstig gehalten. Den Terminus Fremdsozialisation hat ursprünglich Jürgen Zinnecker in seine Überlegungen zur Selbstsozialisation eingeführt. Da geht es zunächst darum, Selbstsozialisation als etwas zu fassen, das im pädagogikfreien Raum stattfindet. Möglicherweise trägt die Rückführung des Begriffs in diese Kontexte etwas zur Lösung seiner innewohnenden Problematik bei. Peter Holzwarth plädiert aus diesem G rund dafür, von institutionalisierten Sozialisationsräumen zu sprechen, wobei „informell“ für steht, in denen sich Menschen kulturelle Praktiken und Kompetenzen ohne pädagogische Anleitung aneignen.

Renate Müller betont, dass die Aufnahme des Begriffs der Fremdsozialisation nicht dazu dient, um ihr Konzept dagegen abzugrenzen, sondern „die Idee schwebt im Raum, dass, wenn man von Selbstsozialisation spricht, ja eigentlich auch von Fremdsozialisation sprechen müsste“. Patrick Glogner führt aus, dass der Begriff "Fremdsozialisation" auch aufgenommen wurde, um „Selbstsozialisation“ zu problematisieren und zu reflektieren. Dieses wurde im Artikel als ein Dilemma formuliert und es wurde versucht, einen Ausweg daraus aufzuzeigen, indem die Verhältnisbestimmung als Vorstellung eines Kontinuums angeboten wurde, in dem die beiden Pole (,selbst“ - "fremd“) als Grenzwerte eigentlich nie vorkommen (Stefanie Rhein). Lässt sich das so verstehen, dass die beiden Pole ein Spannungsfeld im Sozialisationsprozess aufbauen, in dem das Verhältnis der beiden Anteile "fremd" und "selbst" immer neu bestimmt werden muss? Patrick Glogner bejaht diese Frage, denn die Formulierung „die Bedingungen spezifizieren, unter denen Selbstsozialisation möglich ist" bedeutet, dass es bestimmte Bedingungen geben muss, unter denen der Prozess den Einschlag in die eine oder andere Richtung nimmt. Im Artikel wurden auch Beispiele konstruiert, in denen deutlich wird, dass informelle Lern- oder / und Aneignungsprozesse vorstellbar sind, in denen eher Fremdsozialisation stattfindet, und institutionalisierte Sozialisationsprozesse, in denen Sozialisationsprozesse selbstorganisierter ablaufen. Dieses unterstützt Petra Reinhard-Hauck durch einen Bericht über Peergruppen-Kontexte, in denen stark fremdbestimmt gehandelt wird, z. B. in bestimmten Jugendcliquen. Auch in institutionalisierten pädagogischen Kontexten kann die Fremdbestimmung mehr oder weniger dominieren. In diesen Kontexten geht es dann darum, die Bedingungen zu benennen, die für eine stärkere Fremd- oder Selbstbestimmung sprechen, dazu zählen z. B. die Eruierung von Verbindlichkeitsgraden, aber auch gesamtgesellschaftliche Mechanismen in demokratischen oder diktatorischen Strukturen.

Übereinstimmend wird verdeutlicht, dass der Einfluss von Sozialisationsagenturen wie Familie, Schule oder Arbeitswelt nicht automatisch Fremdbestimmung bedeutet. Horst Niesyto führt aus, dass der Vermittlungszusammenhang und die Verarbeitung komplexer Vorgaben und Einflüsse auch in bestimmten institutionalisierten Formen bei einzelnen Individuen höchst unterschiedlich verläuft. Denn die Angebote existieren immer nur vom Individuum aus, das als solches Wirk- 
lichkeit konstruiert und zuschreibt. Es ist immer ein Aneignungsprozess, der unterschiedlichen Verarbeitungsstrategien unterliegt, mehr oder weniger bewussten, mehr oder weniger reflektierten, mehr oder weniger emanzipierten. Mit der Differenzierung der Sozialisationsangebote und -agenturen ist allerdings noch keine Aussage darüber gemacht, wie der Sozialisationsprozess selbst abläuft. Auch in diesem Fall hält Horst Niesyto den Begriff der Fremdsozialisation für irreführend, weil das Individuum diesen Prozess durchführt, egal, ob es sich stark anpasst oder ob es in eine andere Richtung geht.

Manfred Pirner versucht eine Parallele zur Bildungstheorie: Lässt sich das Begriffspaar Fremd- und Selbstsozialisation analog zu den Termini objektive Bildung (auf kulturelle Prägung, auf intentionale Bildung bezogen) und subjektive Bildung (als funktionale, nicht-intentionale Bildung) verstehen? Falls das denkbar ist, wäre in diese Verhältnisbestimmung eine nicht-intentionale Bildung viel stärker in Bildungsprozesse einbezogen. Trotz dieser Vorschläge verstärkt sich im Laufe des Diskussionspunktes die Frage, ob der Begniff der Fremdsozialisation nicht eher verdeckt, was mit Selbstsozialisation eigentlich gemeint ist. Ich gehe noch einen Schritt weiter und halte die Gegenüberstellung des Begriffspaars für eine Sackgasse, auch aus folgendem Grund: Geht man davon aus, dass Selbstsozialisation als kritischer Begriff gegen defizitär empfundene klassische Sozialisationstheorien entwickelt wurde - insbesondere werden in dieser Hinsicht im Text kulturelle Hierarchisierung, mangelnde Differenzierungen, gesellschaftliche Dichotomie von Hochkultur und Popkultur angesprochen - verschiebt der Begriff der Fremdsozialisation die kritische Perspektive. Durch die Dichotomisierung entstehen Fragestellungen, die gar nicht die (als defizitär beschriebenen) Inhalte betreffen, aus deren Gründen diese Theorie erwachsen ist.

\section{Identität - Autonomie des Sub- jekts}

Die Möglichkeit der Autonomie des Subjekts, der freien Wahl des Individuums spielt in der Theorie der Selbstsozialisation eine zentrale Rolle. Manfred Pirner führt aus, dass in der neueren Philosophie der Autonomiebegriff fraglich geworden ist bzw. dass der Gedanke der Autonomie des Subjekts weitgehend aufgegeben wurde. Als Theologe teilt er die tiefe Fragwürdigkeit der eigenen Entscheidung in Glaubensfragen. Auch neurophysiologisch verdichten sich die Erkenntnisse: Es gibt nur den Anschein von Autonomie, es ist „ein Eindruck des Menschen von von sich selber, der sich als Illusion erweist". Holen die Soziologie und Pädagogik den Autonomiebegriff relativ unreflektiert $\mathrm{zu}$ rück? Es ist die Frage, wie man in dieser Situation mit der starken Subjektbetonung umgeht. Es gibt nach wie vor starke Faktoren wie Prägung, Bestimmung von außen, Eingebettetsein in bestimmte Kulturen, die die freie Wahl einschränken.

In der Theorie der Selbstsozialisation ist der Identitätsbegriff auf die Subjektfrage zugespitzt. Horst Niesyto fragt, was vor diesem Hintergrund „Balancierte Präsentation seiner Identität" bedeutet. Es geht um selbst gewählte Themen, um Sinnmärkte. Am Beispiel „Breakdance“ und „Mode“ wird diskutiert, wie Wahlentscheidungen entstehen, mit welchen unterschiedlichen Niveaus von Bewusstheit gewählt wird und wie die komplexen Aneignungsprozesse verlaufen. Es gibt berechtigte Zweifel, ob man das immer als klare, bewusste Wahl bezeichnen kann. So manches Mal erweist sich die selbstbewusst propagierte Autonomie des Subjekts als Illusion. Die Frage ist auch, welche Art, Intensität und Formen der D istanz und Reflexivität wir heute antreffen. Manfred Pirner fragt provokativ im Jargon der kritischen Theorie: „Ist unsere Konsumideologie nicht so weit, dass sie die Massen gleichschaltet, bewusst mit Modetrends überschwemmt und ihnen gleichzeitig vermittelt: Du bist einzigartig, es ist allein deine Entscheidung, du handelst autonom. Das ist eigentlich das Raffinierteste, was es gibt!"

Renate Müller betont, dass es zum einen zentral um die Inhalte der Aneignung geht. Zum anderen entscheidet sich das Individuum selbst für den bestimmten Inhalt und investiert viel Zeit, um etwas zu lernen, von dem es sich entschieden hat, dass es das jetzt lernen will, z. B. Breakdancen. D afür wird in komplexen, langwierigen Aneignungsprozessen Lebenszeit aufgebracht und geopfert. D araus erwachsen pädagogische Konsequenzen: z. B. die Lehrerin unterweist nicht, sondern moderiert einen Lernprozess, in dem Schüler-Experten selbst den Unterricht steuern.

Manfred Pirner ergänzt aus kritischer Sicht weitere bestimmende Faktoren einer subjektbetonenden Sicht der Selbstaneignung und Eigenkompetenz in pädagogischen Räumen. Es gibt einen unterschiedlichen Bedarf an Orientierung, je nachdem, ob ich mich in einer Hauptschulklasse, auf dem Gymnasium oder in einer Klasse mit Lernbehinderten befinde. Wenn man sich Untersuchungen zur Medienrezeption von Kindern anschaut, dann wird oft betont, dass sich die aktive Aneignung von Medieninhalten darin zeigt, dass mediale Bruchstücke in das eigene kind- 
liche Selbstverständnis eingebaut werden. Dieser Prozess lässt sich auch anders verstehen: Kleine Kinder haben noch nicht gelernt, die symbolischen Bedeutungen kulturkonform zu interpretieren, sie beherrschen die Sprache noch unvollkommen. Daher haben sie noch eine größere Freiheit, Einzelheiten selbstständiger zu verstehen. Einerseits lässt sich eine innere Entwicklung im Sinne einer steigenden kognitiven Fähigkeit zur Selbstbestimmung und zur Selbstsozialisation beobachten, andererseits existiert eine äußere Entwicklung, die eine zunehmende Eingebundenheit in eine Kultur mit sich bringt. Sowohl das Verständnis vom selbstständigen, sich selbst sozialisierenden Kind als auch vom Kind, das immer mehr von außen bestimmt wird, wird damit konterkariert, denn beide Tendenzen laufen nebeneinander und stehen in Spannung zueinander. Vor diesem Hintergrund ist es fraglich, wie selbstständig und aktiv konstruierend kleine Kinder mit Medieninhalten umgehen und inwiefern das bereits eine Kompetenzleistung ist.

\section{Nomativität}

Die angesprochenen Themen legen nahe, Selbstsozialisation nicht als quasi normativ neutrale, rein heuristische und deskriptiv analytische Theorie zu verstehen. Im Zentrum der Diskussion stehen nach wie vor Fragen nach der ethisch-normativen Wertung in Verbindung mit Kultur- und Medienanalysen, der Bewertung von als problematisch empfundenen Medieninhalten und der inhaltlichen $\mathrm{Be}$ wertung von Aneignungsprozessen. Auch im Zusammenhang von individuell verschiedenen Verarbeitungsstrategien spielt die Normfrage als Frage nach der moralischen Dimension, nach Mündigkeit, Reflexivität und Distanzierungsfähigkeit eine Rolle. G rundbegriffe wie Freiheit und Verantwortung sind auch entscheidend in sozialer Perspektive. Menschen mögen sich zwar sehr aktiv Dinge aneignen, aber dazu gehört auch die Fähigkeit zur Verantwortlichkeit. Zudem stellt sich m. E. die Frage, ob man Jugendlichen in jedem Fall einen Gefallen tut, wenn Lehrerinnen oder Lehrer die selbst angeeigneten und intensiv kultivierten jugendlichen Praktiken Beispiel jugendliche Sprechstile - anerkennen und damit fördem. Es gibt die These, dass die Akzeptanz von jugendkulturellen Praktiken zu mehr sozialer Ungleichheit führen kann, denn das Stützen entsprechender Praktiken fördert die Illusion gesellschaftlicher Anerkennung, die tatsächlich nur sehr reduziert stattfindet. Dagegen wird die Teilhabe an wirklich relevanten gesellschaftlichen Entscheidungen verwehrt.
Für weiteren Diskussionsstoff sorgt der Kritikpunkt „Hierarchisierung / G leichberechtigung von Kulturen". An welchem Punkt stellt sich die normative Frage? Nicht alle Kulturen sind gleichberechtigt (Beispiel „rechtsradikale Kulturen"). Jugendliche greifen Inhalte auf, die nicht im Sinne ihrer Emanzipation und Persönlichkeitsbildung sind. D er Begriff kann in diesem Fall affirmativ in Bezug auf die herrschenden Gesellschaftsstrukturen wirken, indem er gesellschaftliche Erscheinungsformen z. B. unter dem Label „Populäre Kultur" akzeptiert und zu wenig nachfragt, wo in diesen populärkulturellen Erscheinungsformen Strukturen von Macht und Herrschaft implizit wirken (Horst Niesyto). D er Einwand, das Populärkulturelle werde im Text zu wenig kritisch und reflexiv verarbeitet, fordert mich zu der Frage heraus, ob es nicht vielleicht eine der Stärken des Selbstsozialisationskonzepts ist, dass die normativen O rientierungen, die in klassischen Sozialisations- und Bildungstheorien so stark präsent sind, in diesem Konzept zurückgenommen sind, als bewusster Verzicht auf den moralischen Zeigefinger. Es handelt sich dabei um eine Verschiebung der Perspektiven, indem nicht von vormherein über Aneignungsprozesse einer heterogenen Masse negativ entschieden wird, sondern kulturelle Praktiken ernst genommen und zunächst nicht bewertet werden. Demgegenüber betont Manfred Pirner die Notwendigkeit und den Fakt normativer Aussagen. Denn auch die Polaritäten Dogmatismus - Relativismus beschreiben eine Skala mit zwei Punkten, zwischen denen wir uns einpendeln müssen. D ie Textpassage „Keine gesellschaftliche Instanz kann für sich beanspruchen, die Bewertung von Kulturen objektiv und allgemeingültig vorzunehmen" findet seine $\mathrm{Zu}$ stimmung, das heißt aber nicht, dass eine $\mathrm{Ge}$ sellschaft ohne normative, subjektive Kriterien auskommt. Diese Kriterien, z. B. der Lebensförderlichkeit oder der Q ualität, sind immer gewordene, relative, subjektive Kriterien. Auch die Pädagogik kommt nicht umhin, mit normativen Kriterien den Erziehungsprozess zu begleiten.

\section{Selbstsozialisation als kritischer Begriff}

Neben einer heuristischen Komponente der Theorie der Selbstsozialisation, die die subjektorientierten Faktoren im Aneignungs- und Gestaltungsprozess akzentuieren, skizziert Manfred Pirner eine mögliche kritische Perspektive des Begriffs aus bildungstheoretischer Sicht. D abei geht es darum, die Analyse und Spezifikation der Bedingungen, unter denen Selbstsozialisation mehr oder weniger 
möglich ist, zu den Verarbeitungsstrategien des Individuums, die eher Selbst- oder Fremdsozialisation fördern, in Beziehung zu setzen. Bildungstheoretisch ausgedrückt ließe sich auch sagen: die eher die Bildung des Subjekts befördem als seine Manipulation. Das wäre ein angewandter, kritischer Bildungsbegriff oder eine angewandte Kategorie der Selbstsozialisation, wodurch den Schülem mehr eigene Entscheidungsfähigkeit ermöglicht wird. In der Religionsdidaktik wird das unter „Hermeneutik der Aneignung" diskutiert: Lange wurde von der „Hermeneutik der Vermittlung“ gesprochen, jetzt lautet die Fragestellung anders akzentuiert: Wie eignet sich ein Individuum an, was es aufnimmt? und nicht: Wie wird der $\mathrm{Ge}$ genstand vermittelt? Diese andere Wahmehmungsperspektive beinhaltet eine kritische Komponente, die voraussetzt, dass ein gewisser Prozentsatz von Selbstsozialisation möglich sein sollte. Insofern wäre der Selbstsozialisationsbegriff ein kritischer Begriff, was gleichzeitig die Frage aufwirft, wie sich diese Kritik ausrichtet. Patrick Glogner zitiert Umberto Ecos Titel „Apokalyptiker und Integrierte. Zur kritischen Kritik der Massenkultur" als eine Wurzel ihres Konzepts, das die Frage „Wie gehen Menschen mit Kultur und Medien um ?" nicht aus der massifikationstheoretischen Perspektive, sondern aus einer rezipientenorientierten G egenposition heraus formuliert.

Beobachtungen, die aktive Aneignungsprozesse in den Blick nehmen, wenden sich berechtigterweise gegen die "NürnbergerTrichter-Theorie des Konstrukts des passiven Konsumenten" (Horst Niesyto). Es ist richtig, dieses Konstrukt zu skandalisieren. D azu müssen Bedingungen spezifiziert werden, die die Chancen zur Selbstsozialisation eingrenzen, sie möglich machen. $\mathrm{Zu}$ fragen ist, was im Zusammenhang der Aneignungsprozesse an Übertragungs- und Identifikationsprozessen unterschiedlichster Art abläuft. Hat Selbstsozialisation eine "Schlagseite zu kulturalistischen Theorien, wo kulturelle Faktoren sehr stark betont werden und wo zu wenig die 'Schwerkraft von sozialen Lebenslagen und damit verbundenen Ressourcen herausgearbeitet werden?", wie Horst Niesyto unterstellt? Renate Müller verneint dies, denn die Frage nach Macht und Herrschaft ist Bestandteil ihrer Arbeiten, und zwar insofern, als dass die Definitionsmacht dessen kritisiert wird, was an Kultur beispielsweise in Bildungsinstitutionen vermittelt werden soll. Dem liegt u. a. Bourdieus These zugrunde, dass die Hierarchisierung von Kultur nicht unabhängig von der Produktion und Reproduktion sozialer Ungleichheit in der G esellschaft zu sehen ist.
Welche Inhalte, welche Gegenstände werden im aktiven Umgang mit der kulturellen Umwelt angeeignet, auch im Sinne einer weitgehenden Übernahme? Werden problematische Inhalte in der Theorie nicht ausgeblendet? Horst Niesyto gibt zu bedenken, dass das Selbstsozialisationstheorem $\mathrm{zu}$ einem Stück Ideologie werden kann, in dem es keine kritische Auseinandersetzung mit z. B. problematischen Identifikationsvorgängen, wie sie in bestimmten Fan-Kulturen anzutreffen sind, eingeht. Renate Müller antwortet darauf mit einigen Ausführungen zu ihren Forschungen zu Skinhead-Musik. Die leitende Fragestellung lautete: Ist es die Musik, die die Leute gewalttätig macht? Die Auseinandersetzung damit hat nicht zu einer Kritik an den Inhalten, sondern zur Kritik an der Gesellschaft geführt, die bewirkt, dass Jugendliche sich von diesen Inhalten angezogen fühlen und sich damit selbstsozialisieren. Auch vor diesem Hintergrund macht das Konzept der Selbstsozialisation Pädagogik nicht überflüssig, es gibt höchstens eine Befürchtung von bestimmten Seiten, dass es sie überflüssig machen könnte. Selbstsozialisation setzt einen kritischen Akzent gegen eine Pädagogik, die sich immer selbstbewusst stark normativ artikuliert hat und den Jugendlichen wenig Freiraum zugestanden hat. Wichtig ist, diesen kritischen Impuls zu erhalten. Das Konzept der Selbstsozialisation fordert die Sozialisationstheorie kritisch heraus, regt zu produktiven Fragestellungen und zur kritischen Auseinandersetzung an. In dieser Offenheit und in dieser Unterschiedlichkeit der Einschätzungen beschloss Manfred Pirner die D iskussionsrunde.

Besonderer Dank an die Autoren, die sich der kontroversen D iskussion gestellt haben!

Müller, Renate / Rhein, Stefanie / Glogner, Patrick (2004): Das Konzept musikalischer und medialer Selbstsozialisation - widersprüchlich, trivial, überflüssig? In: Hoffmann, Dagmar / Merkens, Hans (Hg.): Jugendsoziologische Sozialisationstheorie. Impulse für die Jugendforschung. Weinheim und München: Juventa, 237 - 252.

\section{Prof. Dr. phil. Peter Imort}

Professor für Musik an der PH Ludwigsburg

A rbeitsschwerpunk te:

Kulturwissenschaftlich orientiert Musikund Medienforschung, Digitale Musiktechnologien in der Populären Musik, Musikunterricht und Neue Medien, Forschungsprojekt „Musikvideo-Produktion in der Schule". 
Kritische Anmerkungen zum Konzept „medialer Selbstsozialisation"

\section{HORST NIESYTO}

Das Konzept medialer Selbstsozialisation (zusammenfassend: Müller u. a. 2004) akzentuiert die E igenleistungen der Individuen im Sozialisationsprozess. Diese Eigenleistungen haben im Laufe der letzten zwei Jahrzehnte zweifelsohne zugenommen. Hintergrund sind die Prozesse gesellschaftlicher Pluralisierung und Individualisierung, die erheblich mehr Möglichkeiten für die Individuen zur eigenen Lebensgestaltung bieten, aber auch mit einer Vielzahl von Aufgaben bezüglich der eigenen Lebensbewältigung verbunden sind. In diesem Zusammenhang haben sich Medien zu einem bedeutenden Sozialisationsfaktor entwickelt, der gerade für Kinder und Jugendliche vielfältige und attraktive Angebote offeriert, nicht zuletzt im Hinblick auf identitätsstiftende Orientierungen. Es lässt sich belegen, dass Prozesse kultureller Freisetzung bei Kindern und Jugendlichen zugenommen haben, also die Lockerung von sozialintegrativen Kontroll- und Filtermechanismen im kulturellen Bereich - die Medien spielen hier eine große Bedeutung. Meine Bedenken gegenüber dem Konzept medialer Selbst-Sozialisation beziehen sich nicht auf diesen analytischen Kem, sondem auf die begriffliche Fassung dieser Prozesse sowie auf bestimmte Theoreme bzw. Annahmen, die mit dem Konzept medialer SelbstSozialisation verbunden sind.

1.

Der Sozialisationsbegriff umfasst schon immer beide Seiten: die gesellschaftlichen Sozialisationsfelder und -einflüsse und die Verarbeitung dieser Einflüsse durch die Individuen. Anders formuliert: Sozialisation umfasst genuin Prozesse der subjektiven Aneignung, der Eigenaktivität der Individuen. Als D oppelprozess von Assimilation und Akkomodation (Piaget) kann Sozialisation als ein aktiver Anpassungs- und Auseinandersetzungsprozess des Individuums verstanden werden. D as Individuum ist in ständiger Bewegung, um vorhandene Strukturen zu erkennen und neue Strukturen aufzubauen. Dieser Prozess ist immer soziales Handeln auf der Grundlage der Bedeutung von Symbolen (der Mensch als „animal symbolicum“, Cassirer). „Daraus folgt, dass Menschen grundsätzlich nicht einer Welt oder Umwelt gegenüberstehen, sondern in einer Welt handeln" (Kron 1996: 152).

D er Begriff „Selbst-Sozialisation“ verwässert insofern den Sozialisationsbegriff, weil er suggeriert, dass es auch Formen der Sozialisation gibt, bei denen das „Selbst” (das für die Eigenaktivität der Individuen steht) nicht beteiligt ist. Dies ist offensichtlich falsch, es sei denn, man ist Anhänger biologistischer und/ oder extrem manipulationstheoretischer Konzepte.

2.

Um sich diesem Dilemma der „Trivialität“ zu entziehen (Sozialisation ist immer Selbstsozialisation; vgl. Müller u. a. 2004: 246), schlagen die Autor/ innen vor, nach den "Verhältnissen von Fremd- und Selbstsozialisation" $\mathrm{zu}$ fragen, wobei sie klarstellen, dass es „weder reine Selbst- noch reine Fremdsozialisation" gebe (ebd., 247). Trotz dieser Klarstellung scheint es mir problematisch, Begriffe wie "Fremdsozialisation“ und „Selbstsozialisation" zu verwenden. Zweifelsohne gibt es Situationen, in denen Individuen in unterschiedlicher Stärke und Intensität äußeren Faktoren und Einflüssen ausgesetzt sind - bis hin zu extremen Formen der sozialen Kontrolle, der Abhängigkeit, der öffentlichen "Meinungsmache". Es gibt auch Sozialisationstheorien, die in unterschiedlicher Weise z. B. sozial-integrative oder kinder- bzw. jugendkulturelle Sichtweisen akzentuieren. Die Verwendung der Begriffe "selbst" und "fremd" suggeriert jedoch eine D ichotomie zwischen äußeren und inneren Faktoren, die dem komplexen Wechselverhältnis und der Verwobenheit von gesellschaftlichen Angeboten und Strukturmustern einerseits und individuellen Deutungsmustern und symbolischen Verarbeitungsformen andererseits nicht ge recht werden.

3.

Die Verwendung der Begriffe „Selbstsozialisation" und "Fremdsozialisa-tion" deutet zugleich auf (implizite) normative A nnahmen im Konzept der Selbst-Sozialisation hin. Eine dieser Annahmen möchte ich kritisch beleuchten: Das Postulat einer starken A utonomie und $\mathrm{W}$ ahlfähigk eit der Individuen.

So nachvollziehbar und unterstützenswert das Postulat einer starken Autonomie- und Wahlfähigkeit der Individuen im Sinne eines auf Emanzipation orientierten Persönlichkeitsund Gesellschaftsverständnisses ist - diese anthropologisch-normative Orientierung ist nicht mit der empirischen Wirklichkeit gleichzusetzen. Aus Alltagserfahrungen und zahlreichen Studien ist bekannt, dass Wahlfähigkeit in hohem Masse an kulturelle und soziale Ressourcen gebunden ist, die auch in unserer $\mathrm{Ge}$ sellschaft nach wie vor höchst unterschiedlich verteilt sind. Müller u.a. konzedieren dies (vgl. Kap. 2.5 ihres Aufsatzes) und verweisen auf die „Kontextabhängigkeit von Selbstsozialisationspotenzialen verschiedener Kulturen" (2004: 247); explizit nennen sie soziale Kon- 
texte wie die Familie, in die man hineingeboren und die Schulklasse, der man zugeteilt wird (ebd.).

Bei der Qualitätsanalyse dieser Ressourcen grenzen sich die Autoren am Beispiel populärkultureller Angebote von der "Vermassungsthese" ab und bezeichnen den "aktiven U mgang" der Individuen mit ihrer kulturellen Umwelt als entscheidendes Kriterium für selbst-sozialisierendes Handeln (ebd., S. 244 f.). Hier wird eine normative Setzung deutlich, die auf kultur- und medienkritische Analysen (weitgehend) verzichtet und G efahr läuft, allein den A kt der individuellen Wahl zum Kriterium für selbstständiges und selbstbewusstes Handeln zu machen. Es war eine der zentralen Intentionen der sog. „Kritischen Theorie“, die Macht der ökonomisch Stärksten aufzuzeigen: wie es der kapitalistischen Ö konomie unter Nutzung technischer Rationalität gelingt, ihre Herrschaft mittels kulturindustrieller Güter ideologisch abzusichern. Dass Adorno und andere dabei übers Ziel hinausschossen und totalitäre Standardisierungen unterstellten verbunden mit einer bürgerlich-elitären Abwertung und Diskriminierung populärer kultureller Ausdrucksformen - ist eine Sache. Es bleibt jedoch die Aufgabe, sich kritisch mit gesellschaftlichen Symbolangeboten und Ressourcen auseinanderzusetzen und in symbolischen Verarbeitungsweisen von Individuen auch problematische Aspekte zu benennen. Herrschaftsinteressen können gesellschaftliche Verhältnisse, Symbolsysteme und Kommunikationsstrukturen produzieren, die z. B. ein mangelndes D enken in $\mathrm{Zu}$ sammenhängen, mangelnde Selbstreflexion, soziale Isolierung befördern. Allein Kriterien wie „aktiver Umgang“ und "individuelle Wahl aus vorhandenen Sinnmärkten" zu nennen, läuft $\mathrm{Gefahr,} \mathrm{affirmativ} \mathrm{zu} \mathrm{argumen-}$ tieren und vorhandene Entfremdungspotenziale (und damit verbundene Machtinteressen) zu übergehen. Gerade im Hinblick auf Identitätsbildungsprozesse ist dieser Punkt wichtig. D ie Fähigkeit zur „,balancierten Präsentation“ von Identität (Müller u. a., S. 239) setzt Selbstreflexion und Erfahrungslemen voraus. Genau dies ist jedoch durch aktuelle gesellschaftliche und (medien-)kulturelle Prozesse bedroht: ein relevanter Teil des massenmedialen Angebots trägt zur Fragmentierung von Wissen und Bewusstsein bei, destruiert ein Denken in Zusammenhängen und ein Erfahrungslernen, das sich nicht im wechselnden und flexiblen „Mitgliedwerden“ in verschiedenen soziokulturellen Kontexten erschöpft.

Entgegen einer dichotomen Sichtweise, die gesellschaftliche Makrostrukturen und individuelle Verarbeitungsmuster gegenüberstellt, wäre es Aufgabe zeitgemäßer Sozialisationsforschung, die komplexen, expliziten und impliziten Wirk- und Aneignungsmechanismen offen zu legen (vgl. Hoffmann / Merkens) - und dabei problematische Formen der symbolischen Aneignung, der Kommunikation und Interaktion nicht auszusparen.

4.

Im Hinblick auf Pädagogik und Bildung legt das Konzept „Selbst-Sozialisation“ eine Präferenz für selbstorganisiertes und selbstgesteuertes Lernen nahe (vgl. Andeutungen hierzu in Müller u. a., S. 246). Aus Alttagserfahrungen (gerade in Hauptschulmilieus) und Studien (vgl. die Hinweise bei Maurer 2004: 108) ist bekannt, dass gerade sog. lemschwache Schüler/ innen Strukturierungen (Instruktion) benötigen, da sie mit offenen Lernarrangements oft Probleme haben. Beides ist nötig: offene Lernformen wie Handlungsorientierung, Projektarbeit und selbstorganisiertes, selbstentdeckendes Lernen und strukturierende Angebote seitens der Pädagog/ innen, um individuelle Lernprozesse zu fördern. Auch wird deutlich: das Postulat einer individuellen Wahlfähigkeit ist als grundlegende Orientierung zwar wichtig, ersetzt jedoch nicht strukturierende Hilfen im Kontext einer subjekt- und lebensweltbezogenen Bildungsarbeit. Wissensaneignung, Erfahrungslernen und Identitätsbildung setzen unverzichtbar eine reflexive Auseinandersetzung mit Themen und Personen voraus, die nicht nur "Spass“, „Attraktivität" etc., sondern auch Eigensinniges, Widerständiges, Reibungsflächen bieten. Hierzu bedarf es gezielter Anregungen, Arrangements, Instruktionen - „Selbst-Sozialisation“ reicht nicht aus.

\section{Schlussbemerkung}

Der Begriff „Selbst-Sozialisation“ hat einen gewissen heuristischen Wert: er lenkt die Aufmerksamkeit konsequent auf die Subjektseite im Sozialisationsprozess und betont gegenüber sozial-integrativen Ansätzen die kulturellen Aneignungs- und Gestaltungsprozesse von Kindern und Jugendlichen. Als sozialisationstheoretischer Begriff ist er jedoch aus genannten $\mathrm{G}$ ründen problematisch. Ich hatte ihn im gedanklichen Umfeld der Bielefelder Konferenz (1998) selbst verwendet, bin jedoch zwischenzeitlich zum Ergebnis gekommen, dass der Begriff mehr verwischt, denn wirklich klärt. Es gibt unterschiedliche Sozialisationstheorien, die jeweils entwicklungstheoretische, psychoanalytische, lemtheoretische, handlungstheoretische, systemtheoretische, gesellschaftstheoretische Dimensionen akzentuieren. Die dem Konzept der "Selbst-Sozialiation“ zugrunde liegende Analyse und Intention verstehe ich als 
medienkulturtheoretischen Ansatz - und möchte deshalb empfehlen, nicht von "SelbstSozialisation", sondern von einem „(medien)kulturtheoretischen Sozialisationsmodell" zu sprechen. Die inhaltliche Ausgestaltung eines solchen Modells ist noch weitgehend ein D esiderat der Theoriebildung.

\section{Literatur}

Hoffmann, Dagmar / Merkens, Hans, 2003: Bericht zur Tagung „Sozialisationstheorien auf dem Prüfstand" der Sektion Jugendsoziologie der DGS vom 22.2.-24.2.2003 an der Freien Universität in Berlin. In: Ludwigsburger Beiträge zur Medienpädagogik. O nlineMagazin Nr. 4 (2003): http:// www.phludwigsburg.de/ medien1/ intzent.htm

Kron, Friedrich W., 1996: Grundwissen Pädagogik. 5. Auflage. München/Basel: UTBVerlag.

Maurer, Bjöm, 2004: Migration und interkulturelle Medienarbeit. Beitrag zur medienpädagogischen Konzeption des EUForschungsprojekts CHICAM. Unveröffentlichte D iplomarbeit. Pädagogische Hochschule Ludwigsburg.

Müller, Renate / Rhein, Stefanie / Glogner, Patrick, 2004: D as Konzept musikalischer und medialer Selbstsozialisation widersprüchlich, trivial, überflüssig? In: Hoffmann, Dagmar / Merkens, Hans (Hg.): Jugendsoziologische Sozialisationstheorie. Impulse für die Jugendforschung. Weinheim und München: Juventa, 237-252.

Niesyto, Horst, 2000: Medienpädagogik und soziokulturelle Unterschiede. Eine Studie zur Förderung der aktiven Medienarbeit mit Kindern und Jugendlichen aus bildungsmäßig und sozial benachteiligten Verhältnissen. Baden-Baden/ Ludwigsburg

\section{Anmerkungen}

$1 \mathrm{Vgl}$. in diesem Zusammenhang den Bericht zur Tagung „Sozialisationstheorien auf dem Prüfstand" der Sektion Jugendsoziologie der D GS vom 22.2.-24.2.2003 an der Freien Universität in Berlin. Dagmar Hoffmann und Hans Merkens schreiben zur Frage der Sozialisationstheorie: „Es sollte extensiv über eine effektive Verknüpfung von makrosozialen Strukturen und mikrosozialen Entwicklungsprozessen nachgedacht werden und darüber, wie man diese in Sozialisationstheorien implementieren kann. (... ) Es bestehen aus handlungstheoretischer diffuse Interdependenzen zwischen Gesellschaft, Medien und Individuum, die expliziten und impliziten Wirkmechanismen bzw. Aneignungsprozesse sind allerdings in Bezug auf Sozialisationsverläufe weitgehend unklar."

2 In diesem Zusammenhang sind auch differenzierende pädagogische Konzepte notwendig, um eine nach wie vor vorhandene „Mittelschicht-Lastigkeit" - z. B. in der Medienpädagogik - zu überwinden. Das geschieht nicht im "Selbstlauf", sondern bedarf gezielter Studien und konzeptioneller Arbeit (vgl. hierzu Niesyto 2000 und Maurer 2004).

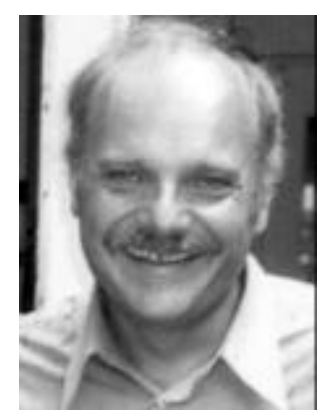

\section{Prof. Dr. Horst Niesyto}

Professor für Erziehungswissenschaft mit Schwerpunkt Medienpädagogik an der $\mathrm{PH}$ Ludwigsburg

A rbeitsschwerpunk te:

Grundfragen der Medienpädagogik; Mediensozialisation; Medienpädagogik und soziokulturelle Unterschiede, schulische und außerschulische Medienpädagogik; handlungsorientierte Medienarbeit (insbesondere Bildmedien); interkulturelle Medienarbeit; Methodenentwicklung in der medienpädagogischen Forschung; medienethnografische Projekte. 


\section{Selbstsozialisation - zur päda- gogischen Tragfähigkeit eines soziologischen Konzepts}

\section{MANFRED PIRNER}

Das Konzept der Selbstsozialisation partizipiert deutlich an dem interdisziplinären Trend zur Akzentuierung des Subjekts und seiner Aktivität, wie er sich im Grunde seit der Neuzeit und noch einmal verstärkt seit Mitte des 20. Jahrhunderts durchsetzt. Wenn ich recht sehe, ist dabei $\mathrm{zu}$ unterscheiden zwischen zwei Aspekten. Der erste ist ein erkenntnistheoretischer und damit anthropologisch-grundlegender G esichtspunkt, der im Gefolge des philosophischen Neukantianismus, des sozialen Interaktionismus, der Kognitionspsychologie und des biologischsystemtheoretischen Konstruktivismus - um nur die vielleicht einflussreichsten Strömungen zu nennen - die konstruktiven und produktiven Leistungen des Individuums in der Auseinandersetzung mit seiner Umwelt herausarbeitet. Bei dem zweiten Aspekt handelt es sich um die Erkenntnis oder These, dass durch Ausdifferenzierung, Pluralisierung und Liberalisierung der westlichen hochindustrialisierten Gesellschaften einerseits immer neue und immer weitere Freiräume für das selbstbestimmte Handeln der Individuen sowie andererseits auch immer umfangreichere Notwendigkeiten zu selbstbestimmtem Handeln und Entscheiden entstehen. Natürlich lässt sich die erkenntnistheoretische Entdeckung des aktiven Individuums noch einmal zurückführen auf die gesellschaftlichen Bedingungen, die diesen Zug des Menschen verstärkt hervortreten, aber auch mit der Brille des Zeitgeistes über die verschiedenen Wissenschaften hinweg verstärkt wahmehmen lassen.

Zum Verständnis und zur Beurteilung (neuer) theoretischer Konzepte erscheint es mir sinnvoll, sowohl deren übergreifende Kontexte als auch deren intertextuelle Verbindungen in den Blick zu nehmen, wie das Müller/ Rhein/ G logner ja selbst ansatzweise tun. Ich werde deshalb in einem ersten Schritt einige Überlegungen zur gegenwärtigen philosophischen Autonomie-Diskussion skizzieren, in einem zweiten Schritt nach soziologisch-sozialphilosophischen Hintergründen fragen, in einem dritten Schritt Parallelen des Konzepts der Selbstsozialisation $\mathrm{zu}$ pädagogischen Perspektiven andeuten und schließlich eine zusammenfassende Bewertung des Konzepts versuchen.

\section{Vom Tod und der Fiktion des Subjekts}

Läuft die Theorie der Selbstsozialisation nicht der Entwicklung in anderen Wissenschaften hinterher und erweist sich damit als eigentlich bereits überholt? Den von Freud so genannten „Kränkungen des menschlichen Selbstbewusstseins" durch die De zentrierung des Menschen aus dem Sternensystem (Kopernikus), aus dem Bereich der Lebewesen (Darwin) und aus seiner eigenen Psyche durch die Entdeckung des nichtverfügbaren Unterbewussten durch Freud sind neue „Kränkungen” hinzugefügt worden: durch die Erkenntnis der gesellschaftlichen Konstruktion von Wirklichkeit, auf die jeder Mensch angewiesen ist und von der er bestimmt ist (Berger/ Luckmann), und neuerdings durch die himphysiologischen $\mathrm{Er}$ kenntnisse, dass das menschliche Gehirn "nichts als" eine komplexe Verschaltung von Nervenzellen darstellt, die durch die Austarierung unterschiedlicher Bedürfnisse gesteuert werden.

D ementsprechend sind in der neueren Philosophie, insbesondere in der französischen (Foucault, Deleuze, Lacan, Derrida, Lyotard), subjekttheoretische Ansätze passé (vgl. Frank u. a. 1988). Hier wird der „Tod des Subjekts" konstatiert. Das Subjekt gilt als eine Fiktion, auch wenn sie als solche vielleicht weiterhin benötigt wird. Eine Selbsterkenntnis des Subjekts im strengen Sinn muss als unmöglich gelten, weil wir uns gleichsam selbst im Wege stehen, wenn wir uns betrachten und bedenken. Darüber hinaus erweist sich die typisch neuzeitliche Unterscheidung von handelndem Subjekt hier und von ihm getrennten Objekten dort als nicht haltbar, wenn erkannt wird, dass der Mensch nicht nur "real”, sondem auch in seiner Wahmehmung, seiner Kommunikation und in seinem Denken vielfältig verwoben ist in vorhandene kulturelle Netze und Texturen. Schließlich ist die subjekttheoretische Sicht auch deshalb unter Kritik geraten, weil mit ihr denknotwendig eine Abwertung gesellschaftlicher Zusammenhänge und sozialer Beziehungen verbunden ist, indem das Subjekt andere zum O bjekt degradiert.

Diese wenigen Andeutungen sollen genügen, um anzuzeigen, dass sich hinter jeglichen subjekt- und autonomie-betonten Ansätzen in Psychologie, Soziologie oder Pädagogik grundlegende Probleme verbergen, die bedacht werden wollen.

Ich sehe das berechtigte Anliegen des Selbstsozialisations-Konzeptes darin, die Autonomie des Subjekts als k ritische Perspek- 
tive in soziologische und pädagogische Diskurse einzubringen. Ähnlich dem - kritischemanzipatorisch verstandenen - Bildungsbegriff verweist es auf problematische Ausblendungen vorhandener Selbstbestimmung bei den Jugendlichen sowie auf problematische Einschränkungen solcher Selbstbestimmung in kulturellen und pädagogischen Kontexten. Allerdings wäre zu fragen, ob und inwieweit im Rahmen jugendlicher Selbstsozialisation ebenso wie im Rahmen pädagogischer Bildungsprozesse ein nichtidealistisches, nicht-aggressives und nichtsolipsistisches Autonomieverständnis erreicht wird, inwieweit also etwa das Bewusstsein des "Sich-verdankens”, des „Eingewobenseins" und der letzten Unverfügbarkeit des eigenen Subjektseins gefördert wird. Damit werden Kriterien benannt, deren Berechtigung unten noch einmal grundsätzlich unter dem Aspekt der Normativität reflektiert werden.

\section{Von der Freiheit und der Qual der Wahl}

D as Konzept der Selbstsozialisation geht, wie oben bereits erwähnt, davon aus, dass mit der Ausdifferenzierung und Pluralisierug unserer Gesellschaft (und dies gerade im Bereich von Medien und Musik) eine "Optionsgesellschaft" entstanden ist, die dem Individuum vermehrt Freiheitsspielräume eröffnet und somit seine Selbstbestimmtheit stärken kann. Dies ist eine berechtigte Sicht auf die Chancen unserer pluralistischen Kultur, aber nur eine Seite der Medaille. Die andere lässt sich mit Peter L. Berger als „Z Zwang zur Häresie" (Berger 1980) beschreiben, d.h. zugespitzt handelt es sich um einen ständigen Zwang, wählen und entscheiden zu müssen, einen Zwang zur Freiheit, der gerade von nicht wenig Jugendlichen auch als Überforderung erlebt werden kann (vgl. dazu auch Bauman 1999). Dass diese Situation psychisch destabilisierend bis krankmachend wirken kann, liegt ebenso auf der Hand, wie dass sie den Hang zu Gemeinschaften, Medienangeboten und (Sub-)Kulturen mit klaren, einfachen Profilen und Regeln, die Orientierung und Halt in der "neuen Unübersichtlichkeit" (Habermas) geben können, verstärken kann.

Die "Gretchenfrage" in Bezug auf das Konzept der Selbstsozialisation wäre vor diesem Hintergrund die, ob und inwieweit es die Fähigkeit von Jugendlichen, selbstbestimmt zu wählen, selbstbestimmt mit Medien umzugehen und selbstbestimmt ihre Identität aufzubauen überschätzt, ob es - anders formuliert - derselben Tendenz unterliegt wie die im Rahmen der Postmodeme-Diskussion immer wieder bemühte Rede von „bricolage”- oder „patchwork”-Identitäten, nunft (Wolfgang Welsch) ihr Leben meistem, so fragmentarisiert und unstet sie sich auch erfahren. Wird hier aber nicht das Idealbild einer reifen, reflektierten, "gebildeten" Persönlichkeit mit hoher Frustrations- und Fragmentierungs-Toleranz gezeichnet, dem nur wenige Menschen und noch weniger Jugendliche in unserer $\mathrm{G}$ esellschaft wirklich entsprechen?

Dennoch: Ich sehe das berechtigte Anliegen des Konzepts der Selbstsozialisation darin, die vorhandenen Kompetenzen der Jugendlichen wahrzunehmen und positiv zu werten. Pädagogische Interventionen werden dabei m. E. aber gerade nicht überflüssig, sondern besonders dringend, denn es geht ja darum, diejenigen Fähigkeiten, die in pluralistischen (Medien-)Kulturen (über-)lebensnotwendig sind, zu stärken und v. a. auch die Chancen der schwächeren und weniger begabten Jugendlichen für ein gelingendes Leben zu vergrößern.

Dabei halte ich es für hilfreich, gerade auch den pädagogischen Blick für humanisierende Tendenzen innerhalb der Jugendkulturen (und ebenso innerhalb der Medienkultur) durch die Perspektive der Selbstsozialisation zu schärfen. So habe ich z. B. beobachtet, dass - entgegen allen Unkenrufen einer immer weiter gehenden Mediatisierung und Technisierung jugendlicher Kommunikationsformen - körperlich-sinnliche Begrüßungsrituale in den letzten Jahren eine wahre Renaissance feiern: vom markigen oder lässigen Händedruck über die Umarmung bis zum zwei- oder dreifachen Küsschen - Körperkontakt bei Begrüßung und Verabschiedung ist hip. Für mich ein Zeichen dafür, dass sich in Jugendkulturen manchmal „von selbst” Umgangsformen und Aktivitäten entwickeln (und durch Selbstsozialisation angeeignet werden), die lebensbeeinträchtigende Einseitigkeiten kompensieren. Und ein Hinweis darauf, dass wir PädagogInnen Jugendlichen hier häufig zu wenig zutrauen.

\section{Von der Norm der nomativen Abstinenz}

Mit der Hauptströmung der Erziehungswissenschaft scheint das Selbstsozialisationskonzept einen gewissen Hang zur normativen Abstinenz, ja geradezu eine Abneigung gegenüber normativen Perspektiven zu teilen. Sie rührt, wenn ich recht sehe, von einer wiederum berechtigten Abwehr vorschneller Bewertungen bzw. Abwertungen von Jugendkulturen und der populären Kultur, wie sich im Schlussteil des Aufsatzes von Müller/ Rhein/ Glogner deutlich zeigt. Dabei ist im Sinne eines aufgeklärten "Norm-Bewusstseins” darauf aufmerksam zu machen, dass auch das Konzept der Selbstsozialisation deutlich nor- 
mative Züge trägt: Es will ja die Selbsttätigkeit und Eigenständigkeit der Jugendlichen und ihrer Kulturen in den Vordergrund rücken und als positiven Wert reklamieren.

Problematisch erscheint es mir jedoch, wenn der G rad der Selbstsozialisation (gegenüber Fremdsozialisation) zur einzigen Norm zur Beurteilung von Sozialisations- und Bildungsprozessen erhoben würde. Dem entspricht die in der Pädagogik manchmal anzutreffende Verabsolutierung von Selbstbestimmung als höchste Norm. So richtig es ist, dass „,keine gesellschaftliche Instanz heute für sich beanspruchen kann, die Bewertung von Kulturen objektiv und allgemeingültig vorzunehmen", und so richtig es ist, dass Werte und Werturteile nicht (mehr) mit dem Anspruch von Objektivität und Absolutheit vorgebracht werden können, so bedenklich wäre es, gesellschaftlich wie pädagogisch, bei einer „begrifflichen Unterscheidung verschiedener Kulturen ohne damit einhergehenden Bewertungen" stehen zu bleiben. Richtig ist sicher, dass eine so genannte Hochkultur nicht per se das Prädikat „pädagogisch wertvoll” verdient, und dass einer so genannten Jugend- oder populäre Kultur nicht pauschal das Stigma „pädagogisch bedenklich” aufgedrückt werden darf. Aber sowohl bezogen auf die „, Hochkultur" als auch auf die „Popularkultur” müssen Kriterien der Lebensförderlichkeit, der Humanität, der ästhetischen Qualität usw. gefunden werden, wenn nicht die Wertfundierung unserer Kultur - z. B. in den Grundwerten und Menschenrechten der westlichen D emokratien generell geleugnet werden soll und wenn nicht das Orientierungsbedürfnis von Jugendlichen frustriert werden soll.

D as Selbstsozialisationskonzept könnte v. a. darauf aufmerksam machen, dass und wie Jugendliche sich selbst in ihrer Lebenswelt soziale und ethische Orientierung suchen und auch finden. Unter pädagogischer Perspektive dürfen die Heranwachsenden nicht als unbeschriebene Blätter, als formungsbedürtige Knetmasse oder schlechthin orientierungslos umhertreibendes Gut am Strand des Lebens angesehen werden. Es geht vielmehr darum, ihre eigenen Orientierungsleistungen und ihre bereits gefundenen Orientierungen zu würdigen und ernst zu nehmen, aber sie eben auch zur Weiterentwicklung und ggf. auch zur Veränderung und Korrektur ihrer Lebensorientierungen anzuregen.

\section{Von der Gleichgültigkeit der un- gleichen Inhalte}

Sieht man sich Beispiele der neueren, rezipientenorientierten Medienforschung an, so betonen die meisten, dass bereits Kinder und erst recht Jugendliche in weit gehend aktiver, konstruktiver, produktiver und souveräner Weise mit den Medien und ihren Inhalten umgehen. Immer wieder wird aufgezeigt, dass sich die RezipientInnen das aus den Medieninhalten heraussuchen, was sie zur Bewältigung ihrer Lebens- und Entwicklungsaufgaben „brauchen können” und solche Bruchstücke sehr eigenständig in ihr Selbst- und Weltverständnis einbauen. D as Konzept der Selbstsozialisation folgt offenbar solchen Untersuchungen.

D abei entsteht manchmal der Eindruck, der Inhalt und die Qualität der Medien sei letztlich relativ gleichgültig, denn auch mit nach herkömmlicher Sicht - problematischen Angeboten könnten Kinder und Jugendliche so umgehen, dass sie zu ihrer Lebensbewältigung und -entwicklung beitragen. Das Konzept der Selbstsozialisation kann dazu helfen, die in der Tat oft erstaunliche Kompetenz der Heranwachsenden wahrzunehmen und schätzen zu lernen, auch aus "dem größten Schrott" von Medieninhalten - wie übrigens auch aus dem unsäglichsten Erziehungsverhalten Erwachsener - etwas Produktives für sie selbst zu machen. Es sollte aber nicht dazu dienen, das Verantwortungsbewusstsein der Erwachsenen für sinnvolles und notwendiges pädagogisches wie politisches Handeln zu schwächen. Weil die Medieninhalte eben nicht gleichgültig sind, wie sich aus anderen Untersuchungen aus dem Bereich der Medienwirkungsforschung lernen lässt, ist politische Einflussnahme ebenso gefragt wie das Bekanntmachen der Heranwachsenden mit alternativen Medienangeboten und die Stärkung ihrer Bewertungs- und Auswahlkompetenzen sowie ihrer Kritikfähigkeit.

Auch hier zeigt sich m. E. noch einmal, dass es letztlich nicht allein um die Frage von Selbstsozialisation oder Fremdsozialisation gehen kann und auch nicht um die Frage, ob „Hochkultur” oder „Popkultur”, sondern es sind vor dem Zielhorizont eines menschenwürdigen Lebens in einer menschenwürdigen Gesellschaft Kriterien in Anschlag zu bringen, welche die Beurteilung von Inhalten, Aneignungs- und Bildungsprozessen erlauben. Als wichtiges Anliegen des Selbstsozialisationskonzepts ist festzuhalten: Auch die populäre Kultur und der jugendliche Umgang mit ihr enthalten „humanes Kapital", tragen Ressourcen und Impulse der Humanität zur individuellen und gesellschaftlichen Entwicklung bei. D eshalb wird es gut sein, Jugendliche und „ihre” Kultur in politischen, gesellschaftlichen, kulturellen und pädagogischen Belangen stärker zu berücksichtigen. 
D ennoch darf auch nicht aus dem Blick geraten, dass populäre Kultur eben auch kommerziell „gemacht” wird, dass sie auch eine Vermassung, Verflachung und Verrohung herbeiführen kann, dass sie Jugendliche auch manipuliert und sie zu Sklaven des Konsums (fremd-)sozialisieren kann. Neben dem aktuellen Phänomen der Handy-Verschuldung (Jugendliche sind $\mathrm{z}$. T. hoch verschuldet, weil sie über dem Reiz des Handy-Telefonierens die Kosten aus den Augen verlieren) scheint mir die Mode nach wie vor ein eindrückliches Beispiel zu sein, wie das $G$ efühl von Freiheit und Selbstbestimmung in manipulativer Weise vorgetäuscht werden: Wenn meine damals 14-jährige Tochter mir mit dem Aufkommen der Bauchfrei-Mode sagte, dass sie unbedingt ein Bauchfrei-Top kaufen wolle, weil ihr das ganz persönlich gefiele, dann lässt sich das als eine gelungen inszenierte Illusion von Selbstbestimmung und persönlichem Geschmack verstehen. Es wird $\mathrm{m}$. E. wichtig sein, bei der Rede von der Selbstsozialisation Jugendlicher für solche Mechanismen kritisch sensibel zu bleiben -- um der Selbstwerdung der Jugendlichen willen.

\section{Literatur}

Bauman, Zygmunt, 1999: Unbehagen in der Postmoderne, Hamburg.

Berger, Peter L., 1980: Der Zwang zur Häresie, Frankfurt a.M.

Frank, Manfred u. a. (Hg.), 1988: Die Frage nach dem Subjekt, Frankfurt a.M.

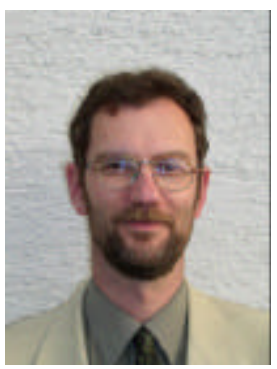

Prof. Dr. Manfred Pimer

Professor für Ev. Theologie / Religionspädagogik an der PH Ludwigsburg

A rbeitsschwerpunk te:

Theologische Hermeneutik und Theorie der Kultur und der Medien, Musik und Religion, Sprachphilosophie und Theologie, Medienpädagogik und Religionspädagogik, Medienerziehung im Religionsunterricht. 